\title{
Testicule et sperme dans le Syndrome de Klinefelter chez l'adulte
}

\author{
ERIC VENDRELY \\ Laboratoire de Biologie de la Fertilité et CECOS, Hôtel-Dieu de Paris, Place du Parvis \\ Notre-Dame, Paris
}

\section{RESUME}

Dans sa forme typique le syndrome de Klinefelter comporte l'azoospermie, l'hypogonadisme hypogonadotrope et souvent une gynécomastie. L'examen microscopique du testicule montre une fibro-hyalinose avec épaississement de la gaine péri-tubulaire et réduction du diamètre des tubes séminifères.

L'épithélium séminal est constitué par les cellules de Sertoli et inconstam. ment par des germinales immatures. On observe exceptionnellement des spermatozoïdes. La glande interstitielle parait hyperplasique, parfois pseudo-adénomateuse. Elle sécrète peu d'hormones stéroïdes.

L'azoospermie est un des signes majeurs du syndrome de Klinefelter mais elle n'est pas constante, parfois limitée à une oligozoospermie sévère. Dans ce cas une certaine fécondité peut être conservée, souvent associée à une constitution chromosomique en mosaïque. Les lésions testiculaires tendent à s'aggraver avec l'âge.

Mots-clés : Klinefelter - Testicule - Sperme Stérilité - Aneuploïdie

\section{INTRODUCTION}

A partir de l'observation de 9 patients stériles KLINEFELTER, REIFENSTEIN et ALBRIGHT [9] ont décrit en 1942 un syndrome apparemment bien défini associant azoospermie, hypogonadisme hypogonadotrope et gynécomastie. Sept biopsies testiculaires montraient une fibro-hyalinose, une spermatogenèse incomplète ou absente selon les sections tubulaires et une hyperplasie leydigienne. Dès cette publication les lésions caractéristiques des gonades et du sperme étaient décrites mais il fallut attendre la découverte de l'origine chromosomique du syndrome pour détailler les anomalies du testicule et du sperme et en décrire les variations. L'étude des biopsies testiculaires en microscopie électronique n'a pas apporté de renseignements complémentaires significatifs $[7,17,19]$.

Le volume testiculaire est habituellement très diminué. Dans une étude portant sur 14 hommes à caryotype $47, \mathrm{XXY}$ et 52 témoins à caryotype normal, Borsen [2] observe une réduction globale de toutes les dimensions et du volume testiculaire.

Les lésions histologiques touchent pratiquement tous les constituants de la gonade. Au microscope, dès le faible grossissement, on remarque la diminution du diamètre tubulaire, l'épaississement de la gaine péri-tubulaire et l'hyperplasie de la glande interstitielle décrits dans la publication initiale (Figure 1).

\section{GAINE PERITUBULAIRE}

Dans le testicule normal la gaine péri-tubulaire a une épaisseur inférieure à $5 \mu \mathrm{m}$. Elle est constituée par la membrane basale très fine de l'épithélium séminifere et une lami- 
na propria conjonctive formée de fibroblastes contractiles (myofibroblastes) riches en actine, entre lesquels sont disposées de fines fibrilles de collagène et d'élastine (Figure 5, 6).

Dans le syndrome de Klinefelter on observe typiquement un accroissement de la quantité de collagène aboutissant à une sclérose progressive de la lamina propria entravant les échanges entre l'épithélium séminifêre et les espaces interstitiels. La finesse des fibres collagènes donne un aspect homogène à cette sclérose qui finit par étouffer complètement le tube séminifère, aboutissant à l'aspect de sclérose tubulaire (Figure 3). Le diamètre tubulaire diminue avec l'épaisseur de la gaine péri-tubulaire mais ce phénomène est plus progressif dans le testicule des sujets 47,XXY que dans les autres causes d'atteinte gonadique [10], l'atrophie des tubes séminifères liée à l'anomalie génétique précédant le développement de la sclérose. La fibro-hyalinose péri-tubulaire s'accompagne dans le syndrome de Klinefelter d'une réduction de la surface des myofibroblastes ce qui n'est pas le cas dans les atteintes testiculaires liées à d'autres origines. L'analyse morphométrique montre que le volume de la gaine péri-tubulaire reste en fait à peu près constant, quel que soit le diamètre tubulaire.

\section{EPITHELIUM SEMINIFERE}

Il est constitué par les cellules de Sertoli et les cellules germinales lorsqu'elles existent. Dans ce cas elles sont disposées en plages évoluant vers une spermatogenèse incomplète (Figure 2). En fait l'aplasie germinale est le plus souvent totale sauf en cas de mosaique 46,XY/47,XXY. GoRDON et coll. [8] ont analysé la constitution chromosomique des tubes séminifères de 12 patients, 6 de constitution $47, \mathrm{XXY}$ homogène et 6 mosaïques. En pratique seuls les patients en mosaïque possédaient un nombre important de tubes séminifères contenant des cellules germinales évoluant jusqu'à un stade avancé de la spermatogenèse.
Les cellules de Sertoli sont souvent seules présentes dans l'épithélium séminifère des sujets atteints de syndrome de Klinefelter. Leur morphologie est caractéristique, avec un noyau profondément encoché, une chromatine très fine, claire et homogène et un volumineux nucléole de structure complexe (Figure 8). Le cytoplasme contient des enclaves de glycogène et de lipides [12].

\section{GLANDE INTERSTITIELLE}

L'hyperplasie de la glande interstitielle est signalée dans la description initiale de $\mathrm{Kli}$ nefelter. Le plus souvent il s'agit en fait d'une augmentation du volume relatif comme l'ont montré AHMAD et coll. [1] par une étude morphométrique couplée à l'évaluation du volume testiculaire. Il existe toutefois de réelles hyperplasies leydigiennes allant jusqu'à donner des aspects pseudoadénomateux en nappe diffuse (Figure 4).

L'étude ultrastructurale [16, 17, 14] montre que les cellules de Leydig sont bien différenciées, avec un réticulum endoplasmique lisse très développé (Figure 7). Cet aspect ne traduit en fait pas une stéroïdogenèse efficace [6]. Les cristalloïdes de Reinke sont très rares et peu volumineux. Les cellules interstitielles indifférenciées, fibroblastoïdes, sont moins nombreuses que dans le testicule normal, ce qui traduirait une accélération de leur différenciation sous l'influence des hormones gonadotropes.

L'hypoplasie leydigienne peut se voir lorsque existe une diminution de la sécrétion des hormones gonadotropes et surtout lorsque la fibro-hyalinose envahit le testicule (Figure 3) [13].

L'azoospermie est un des signes majeurs du syndrome de Klinefelter dans sa forme accomplie. On sait depuis longtemps que ce n'est pas toujours le cas. Selon Futterweit [5] si l'azoospermie est habituelle le syndrome de Klinefelter peut se traduire par une oligozoospermie sévère, le plus souvent inférieure à 1 million de spermatozoïdes par $\mathrm{ml}$ 


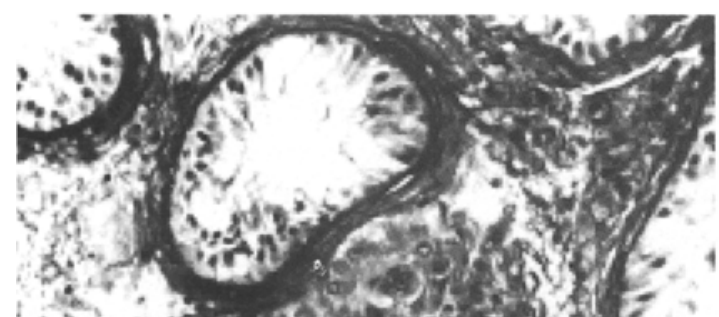

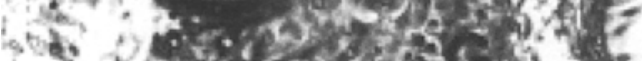

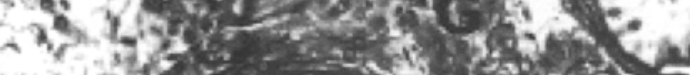

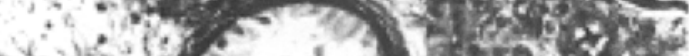

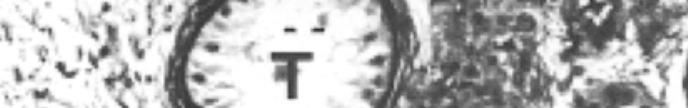

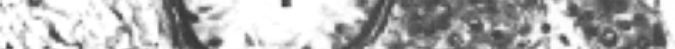

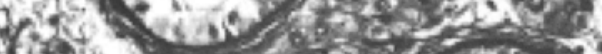

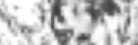

ind

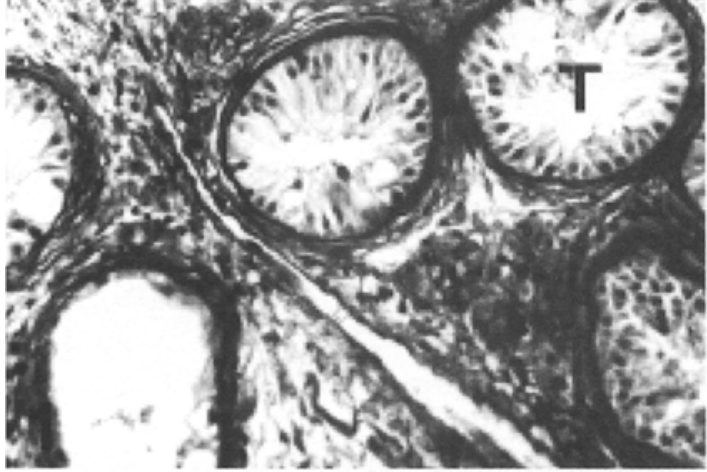

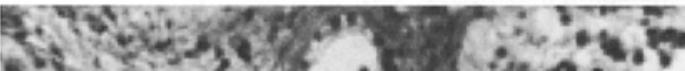

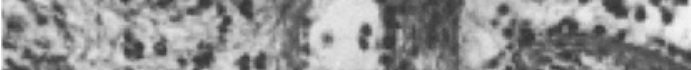
$x^{2},-0.42$.

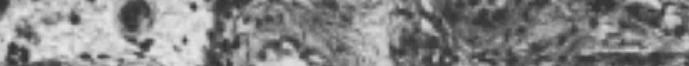
ber.

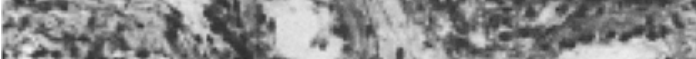

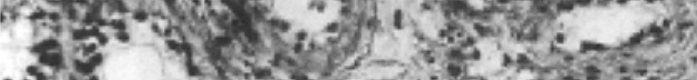

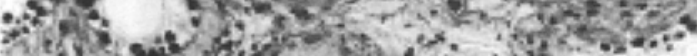

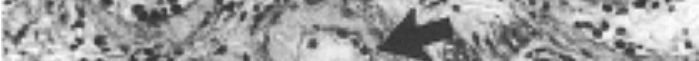

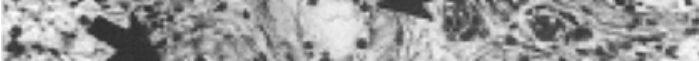

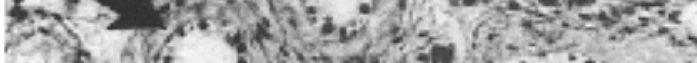

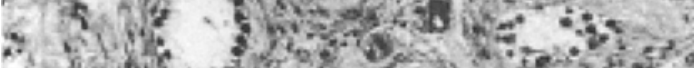

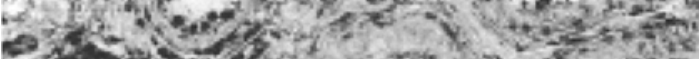

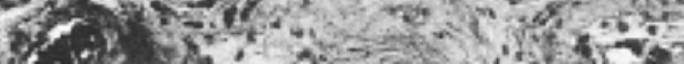

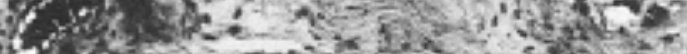
- y t

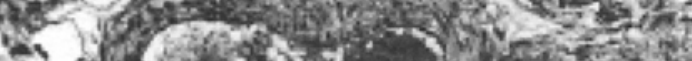

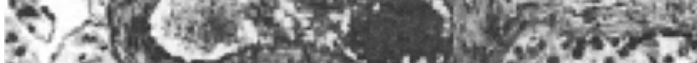

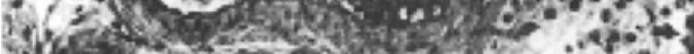
Afin $n$,

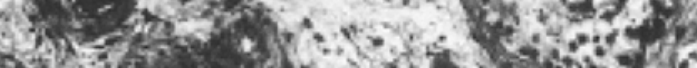

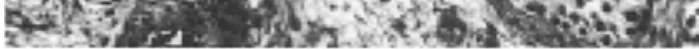

4f.

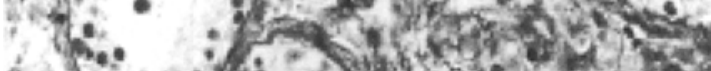

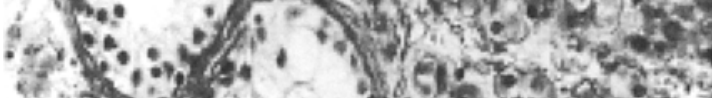

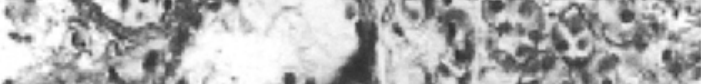

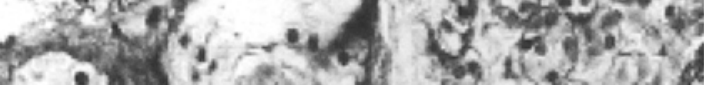

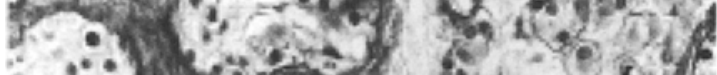

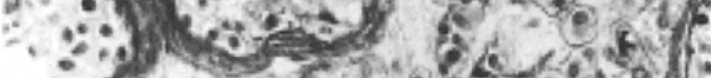

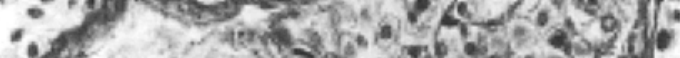

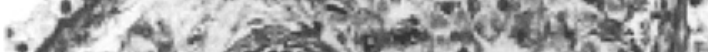

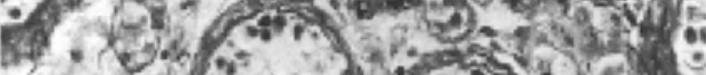

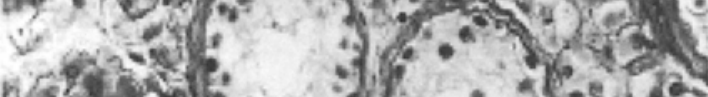

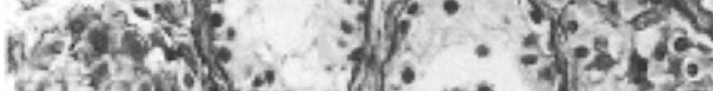

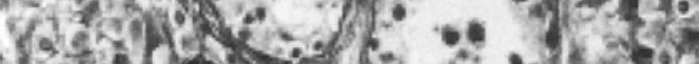

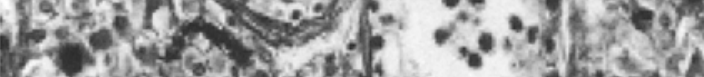

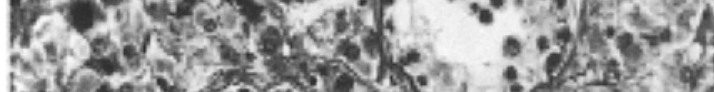
cos 3 \%

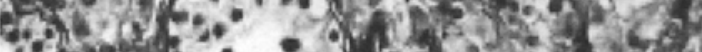

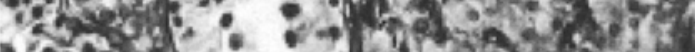

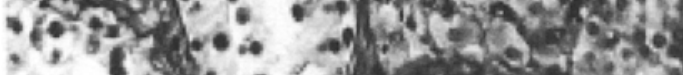
in P(

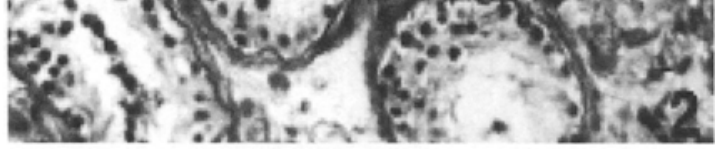

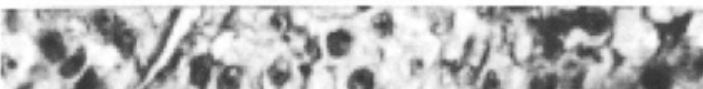

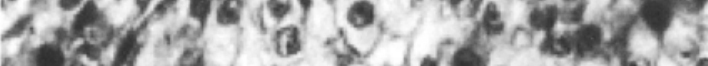

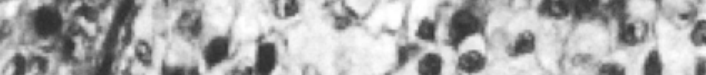

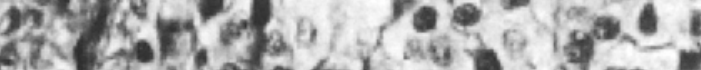

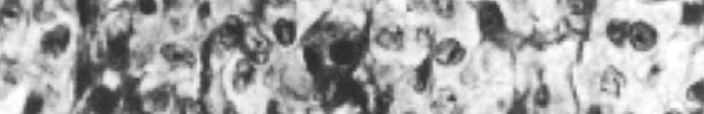

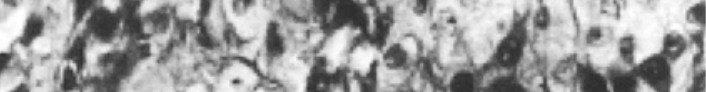

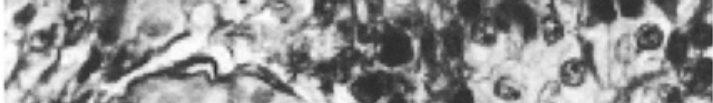

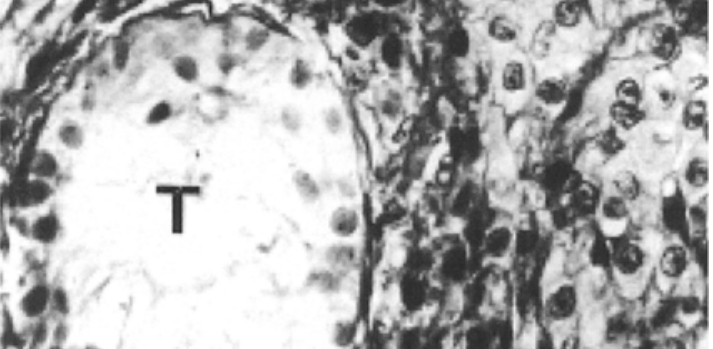

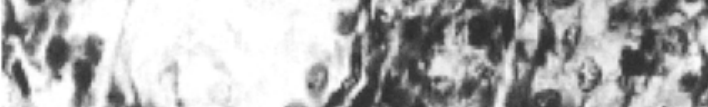

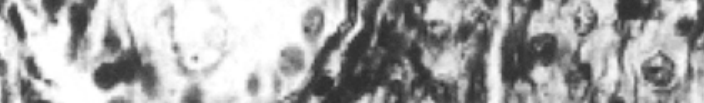

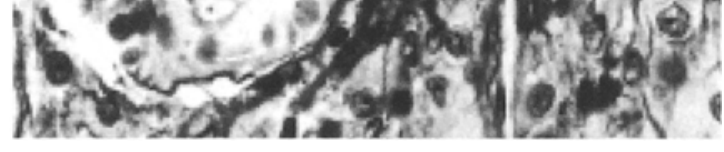


Microscopie photonique de testicule de sujets atteints de syndrome de Klinefelter :

1. Les tubes séminifêres $(T)$ ont un diamètre réduit et ne contiennent que des cellules de Sertoli. La glande interstitielle $(G)$ paraît hyperplasique. $(x 400)$.

2. Les tubes séminifêres, de diamètre réduit et dont la gaine péritubulaire a une épaisseur normale, contiennent parmi les cellules de Sertoli des cellules germinales qui ne dépassent pas le stade de spermatocyte de premier ordre. $(x 400)$.

3. Testicule en voie de hyalinisation tubulaire. Les tubes séminifêres ont un très faible diamètre et ne contiennent que des cellules de Sertoli. La gaine péritubulaire $(\rightarrow)$ est très épaissie et scléreuse. La glande interstitielle, atrophique, est inapparente. (x200).

4. Glande interstitielle hyperplasique pseudo-adénomateuse. Le seul tube séminifêre visible, très atrophique, ne contient que des cellules de Sertoli. (x 800).

mais pouvant atteindre 5 millions par $\mathrm{ml}$. Ceci explique que l'on connaisse des cas à vrai dire peu fréquents de sujets fertiles et justifie l'établissement du caryotype des patients souffrant d'oligozoospermie. Sur 466 patients à sperme anormal étudiés par Foss et LEwIS [4], 15 étaient porteurs d'un caryotype 47 , XXY dont 4 avaient des spermatozoïdes mobiles dans l'éjaculat. Le volume du sperme éjaculé est inférieur à la normale ( $2 \mathrm{ml}$ en moyenne) et on observe des troubles de la coagulation et de la liquéfaction. Ces anomalies paraissent en rapport avec la diminution des sécrétions prostatiques et vésiculaires liées à la chute de la testostéronémie [11].

L'analyse du caryotype des spermatozoïdes a été pratiquée chez un sujet porteur d'une mosaïque $46, \mathrm{XY} / 47, \mathrm{XXY}$ [3]. Il a été observé une fréquence de $0,9 \%$ de spermatozoïdes de formule chromosomique 24 , XY, révélant que les spermatocytes subissent la méiose sans disjonction d'une paire de chromosomes sexuels XY.

\section{DISCUSSION}

Les lésions testiculaires primitives liées à la présence d'un chromosome $\mathrm{X}$ surnuméraire dans la formule chromosomique des sujets atteints de syndrome de Klinefelter concernent avant tout l'épithélium séminifere et plus particulièrement les cellules germinales. Ces anomalies sont très semblables à celles que l'on observe chez les hommes à caryotype 46,XX [15]. Cependant dans ce cas les lésions sont plus intenses en raison de l'absence de gènes favorisant la spermatogenèse apportés par le chromosome Y.

L'évolution des lésions gonadiques au cours de la vie se fait vers une aggravation progressive. SMith et coll. [18] considèrent que les cellules présentes à la naissance dégénèrent avant la puberté puis que les cellules de Sertoli seules présentes dans le testicule adulte disparaissent progressivement alors que progresse la hyalinisation tubulaire. $\mathrm{Ce}$ tableau évolutif est peut-être excessivement schématique car il ne reflète pas la variété des lésions histologiques réellement observées.

De nombreux sujets souffrant du syndrome de Klinefelter sont en fait porteurs d'une mosaïque $46, \mathrm{XY}, / 47, \mathrm{XXY}$. L'intensité de l'atteinte de la spermatogenèse semble dépendre de la répartition et de la localisation des clones atteints. Le diagnostic chromosomique en est difficile et nécessite l'étude de nombreux caryotypes portant sur des lignées cellulaires variées. La fréquence des mosaïques par rapport aux syndromes de Klinefelter homogènes est mal connue mais est plus souvent liée à la présence d'une spermatogenèse complète et de spermatozoïdes dans l'éjaculat. La vérification de la constitution chromosomique pourrait alors avoir une valeur pronostique. 

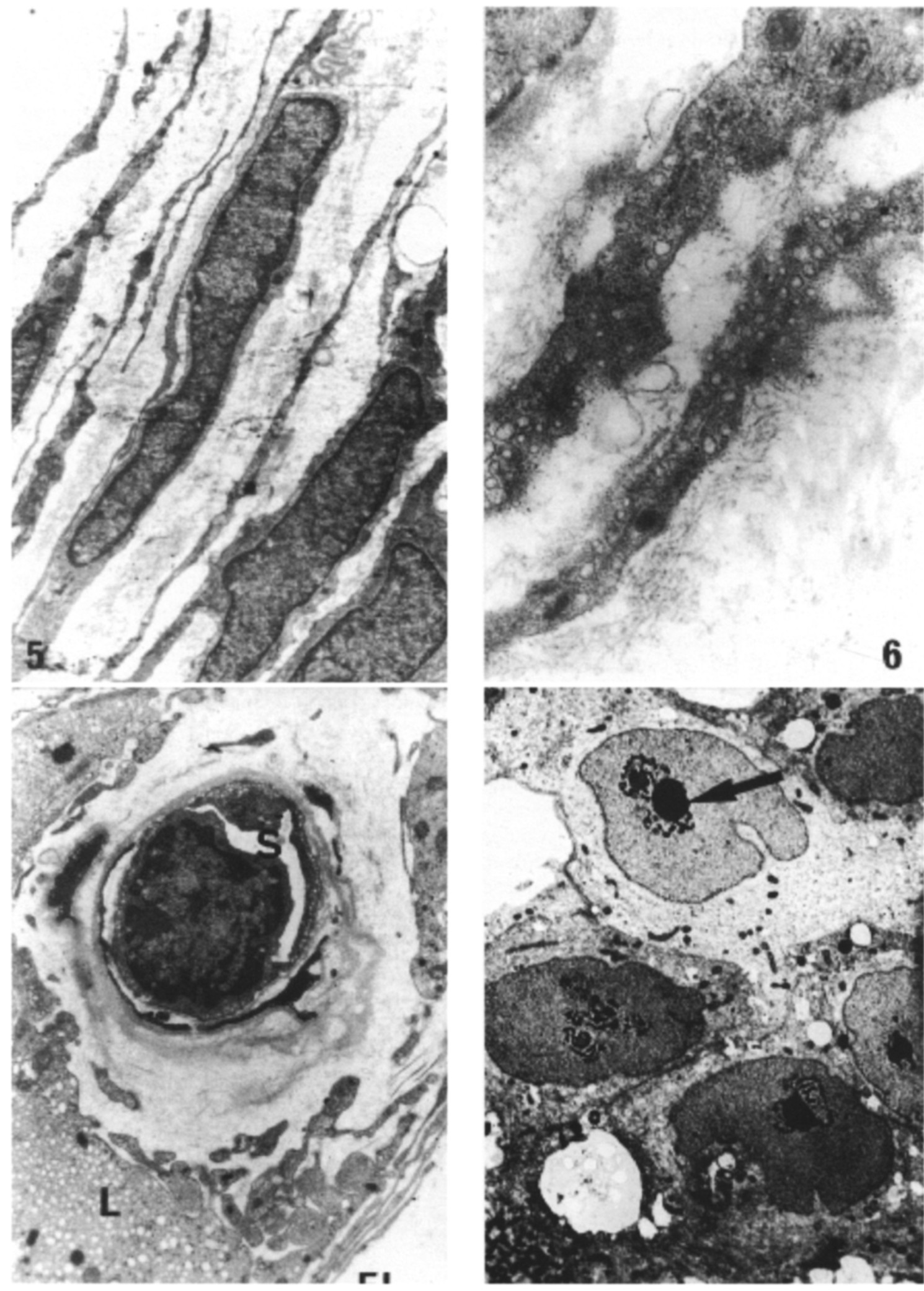

6

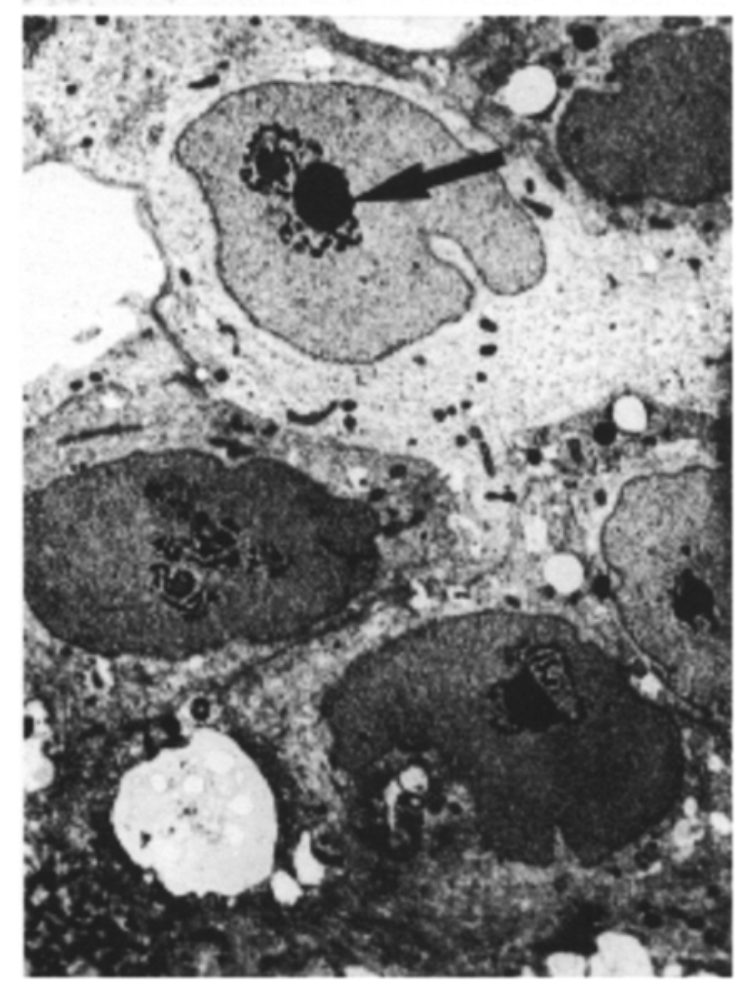




\section{Planche 2: Page de Gauche.}

Microscopie électronique du testicule dans le syndrome de Klinefelter :

5. Gaine péritubulaire en microscopie électronique. Elle comporte de multiples couches de myo-fibroblastes séparés par une abondant matrice extra-cellulaire de fibrilles très fines. $(x 2000)$.

6. Cellules péritubulaires en microscopie électronique. Elles sont riches en vésicules de micropinocytose. $(x$ 10000).

7. Cellules de Leydig ( $L$ ) en microscopie électronique près d'un capillaire sanguin (S) et d'un espace lymphatique $(E L)$. Le reticulum endoplasmique des cellules de Leydig est très développé et les cellules paraissent actives. ( $x$ 7000).

8. Cellules de Sertoli en microscopie électronique. On distingue des cellules claires et des cellules sombres. Le noyau, régulier, contient un nucléole très volumineux et de structure complexe. Le cytoplasme est pauvre en organites. L'épithélium est dépourvu de cellules germinales. (x 1500).

\section{REFERENCES}

1. AHMAD K.N., DYKES J.R., FERGUSON-SMITH M.A., LENNOX B., MACK W.S. : Leydig cell volume in chromatin-positive Klinefelter's syndrome. J. clin Endocr. Metabol., 1971, 33 : 517-520.

2. BOISEN E. : Testicular size and shape of $47, \mathrm{XYY}$ and $47, \mathrm{XXY}$ men in a double-blind, double-matched population survey. Amer. J. hum. Genet., 1979, $31: 697-703$.

3. COZZI J., CHEVRET E., ROUSSEAUX S., PELLETIER R., BENITZ V., JALBERT H., SELE B. : Achievement of meiosis in XXY germ cells: study of 543 sperm karyotypes from an XY/XXY mosaic patient. Hum. Genet., 1994, $93: 32-34$.

4. FOSS G.L., LEWIS F.J. : A study of four cases with Klinefelter's syndrome, showing motile spermatozoa in their ejaculates. J. Reprod. Fertil., 1971, 25 : 401-408.

5. FUTTERWEIT W. : Spermatozoa in seminal fluid of a patient with Klinefelter's syndrome. Fertil. Steril., 1967, 18: 492-496.

6. GABRILOVE J.L., FREIBERG E.K., NICOLIS G.L. : Testicular function in Klinefelter's syndrome. J. Urol., 1980, $124: 825-826$.

7. GOMEZ-ACEBO J., PARRILLA R., ABRISQUETA J.A., POZUELO V. : Fine structure of spermatogenesis in Klinefelter's syndrome. J. clin. Endocr. Metabol., 1968, 28 : 1287-1294

8. GORDON D.L., KRMPOTIC E., THOMAS W., GANDY H.M., PAULSEN C.A. : Pathologic testicular findings in Klinefelter's syndrome. Arch. intern. Med., 1972, 130 : 726-729.

9. KLINEFELTER H.F., Jr, REIFENSTEIN E.C., ALBRIGHT F. : Syndrome characterized by gynecomastia, aspermatogenesis without a-Leydigism, and increased excretion of follicle stimulating hormone. J. clin. Endocr., 1942, 2 : 615627.
10. MARTIN R., SANTAMARIA L., NISTAL M., FRAILE B., PANIAGUA ( $R$. : The peritubular myofibroblasts in the testes from normal men and men with Klinefelter's syndrome. A quantitative, ultrastructural and immunohistochemical study. J. Pathol., 1992, $168: 59-66$.

11. MOON K.H., OSBORN R.H., YANNONE M.E., BUNGE R.G. : Seminal fructose and hormonal studies in Klinefelter's syndrome. Investig. Urol., $1968,6: 179-182$.

12. NISTAL M., PANIAGUA R., ABAURREA M.A., SANTAMARIA L. : Hyperplasia and the immature appearance of Sertoli cells in primary testicular disorders. Human Pathol., 1982, 13 : 3-12.

13. NISTAL M., PANIAGUA R., ABAURREA M.A. PALLARDO L.F. : 47,XXY Klinefelter's syndrome with low FSH and LH levels and absence of Leydig cells. Andrologia, 1980, $12: 426-433$.

14. NISTAL M., SANTAMARIA L., PANIAGUA R. Quantitative and ultrastructural study of Leydig cells in Klinefelter's syndrome. J. Pathol., 1985, 146 : 323-331.

15. ROMANI F., TERQUEM A., DADOUNE J.P. : Le testicule chez un homme XX. A propos d'une étude ultrastructurale. J. Gynecol. Obst. Biol. Reprod., 1977, $6: 1049-1059$.

16. RUBIN P., MATTEI A., CESARINI J.P., LAFFARGUE P., VAGUE J. : Electron microscope study of the Leydig cell in the Klinefelter's disease in pre-, per- and post-pubertal periods. Ann. Endocr., 1971, $32: 671-687$.

17. SABA P., GAMBASSI G., NOVI A.M., LUISI M..Electron microscopy of the Leydig cells and hormone assays in Klinefelter's syndrome. Endokrinologie, $1969,55: 129-144$.

18. SMITH B.D., LEESON C.R., RUNGE R.G. : Microscopic appearance of the testis in Klinefelter's syndrome before and after suppression of gonadotrophin production with testosterone. Investig. Urol., 1967, $5: 58-72$. 
19. SODERSTROM K.O. : Ultrastructure of the testis in Klinefelter's syndrome. Arch. Androl., 1984,13 : 113-119.

\section{ABSTRACT}

Testis and semen in Klinefelter's syndrome in adult

\section{E. VENDRELY}

Klinefelter's syndrome is characterised by azoospermia, gynecomastia and hypogonadotropic hypogonadism. The testes are reduced in volume. Microscopically, seminiferous tubules show a reduced diameter and a thickening of peritubular sheath. The seminiferous epithelium contains Sertoli cells with a clear typical nucleus and a heterogeneous nucleolus. Usually there are no germ cells. When present they degenerate at the spermatocyte stage. Very few achieve a complete maturation until the spermatozoon form.

The interstitial gland appears relatively hypertrophied, due to the decrease in volume in the other components of the testis. Sometimes Leydig cells display an adenomatous morphology. Therefore the steroidogenic function is impaired.

Azoospermia is a major event in Klinefelter's syndrome but sometimes restricted to severe oligozoospermia. In that cases, fertility may be somewhat preserved, and the caryotype usually reveals a mosaicism, 47,XY/47,XXY with a better prognosis than in pure syndrome.

Key-words : Klinefelter's syndrome - Testis Semen - Sterility - Aneuploidy 